\title{
El ecosistema educativo universitario impactado por las $\mathrm{TIC}^{\star}$
}

\author{
Andrés Barrios Rubio** \\ Gloria Consuelo Fajardo Valencia ${ }^{* \star \star}$ \\ Recibido: 2016-08-21 Enviado a pares: 2016-08-16 \\ Aprobado por pares: 2016-09-14 Aceptado: 2016-11-11 \\ DOI: 10.22395/angr.v15n30a5
}

\begin{abstract}
Resumen
El ecosistema digital que impacta a la sociedad de hoy, panorama del que no es ajena la academia, lleva a interrogarse por la necesidad que tienen los docentes de integrar el uso de las TIC en el proceso de formación como apoyo a la presencialidad y por el modelo de educación en el que se inserta este uso. Para abordar este fenómeno se estructuró una investigación de metodología mixta (cuantitativa-encuestacualitativa-entrevista-), aplicada a estudiantes y docentes de Comunicación SocialPeriodismo, desde la cual se abordó un ejercicio exploratorio de praxis comunicativa, sobre el uso de las nuevas tecnologías en el aula, así como la concepción de este uso por parte de los actores involucrados en el proceso formativo. El corpus de análisis permitió evidenciar que en el modelo presencial lo tecnológico se convierte en un apoyo docente, una herramienta, un complemento, por así decirlo, al proceso de enseñanza aprendizaje. El modelo inminentemente virtual o de educación a distancia (e-learning y blended) se plantea como un paradigma de la didáctica tecnológica.
\end{abstract}

Palabras clave: TIC, aula, educación, universidad, comunicación.

El artículo es resultado de la investigación realizada por el grupo Comunicación-Medicación-Cultura de la Universidad de Bogotá Jorge Tadeo Lozano, clasificado en Colciencias, a través del proyecto Uso y consumo de las TIC; y el trabajo de grado de Maestría en Educación "Apropiación de las TIC: concepción, uso, relaciones, prácticas y diseños en el aula (Caso programa Comunicación Social-Periodismo de la Universidad Jorge Tadeo Lozano)", realizado por la investigadora Gloria Consuelo Fajardo Valencia en la Universidad Santo Tomás. COLOMBIA.

* Doctor en Contenidos de Comunicación en la Era Digital, magíster en Administración de Empresas con Especialidad en Dirección de Proyectos, comunicador social-periodista, especialista en Edición Digital y Multimedia. Actualmente, director Emisora Óyeme UJTL y Docente Titular del Departamento de Comunicación Social y Cinematografía de la Facultad de Ciencias Sociales de la Universidad de Bogotá Jorge Tadeo Lozano. andres.barrios@utadeo.edu.co

*** Magíster en Educación, comunicadora social con profundización en Comunicación Organizacional. Actualmente, docente Asociada II del Departamento de Comunicación Social y Cinematografía de la Facultad de Ciencias Sociales, de la Universidad de Bogotá Jorge Tadeo Lozano. gloria.fajardo@utadeo.edu.co 


\title{
The university education ecosystem impacted by ICT
}

\begin{abstract}
Abstrac
The digital ecosystem that impacts society today, a panorama that is not alien to academia, leads to questioning the need for teachers to integrate the use of ICT in the training process as a support for the presence and for the Model of education in which this use is inserted. To address this phenomenon, an investigation of mixed methodology (quantitative-poll-qualitative-interview-) was structured, applied to students and teachers of Social Communication-Journalism, from which an exploratory exercise of communicative praxis was discussed, on the use of New technologies in the classroom, as well as the conception of this use by the actors involved in the training process. The corpus of analysis allowed to show that in the face-to-face model the technological becomes a teaching support, a tool, a complement, so to speak, to the teaching-learning process. The imminently virtual model or distance education (e-learning and blended) is proposed as a paradigm of technological didactics.
\end{abstract}

Keywords: TIC, classroom, education, college, communication.

\section{O ecossistema educacional universitário impactado pelas TIC}

\begin{abstract}
Resumo
O ecossistema digital que o cenário hoje a sociedade impacta não está fora da academia, levanta questões sobre a necessidade de professores para integrar a utilização das TIC no processo de formação para apoiar o presencial idade e modelo de educação no qual se insere este uso. Jornalismo, a partir do qual um exercício exploratório das práxis comunicativas abordadas sobre o uso de - para abordar o fenómeno da metodologia de investigação mista (quantitativa -votação- qualitativa -entrevista-) aplicada a alunos e professores de Comunicação Social foi estruturado novas tecnologias na sala de aula, bem como a concepção desta utilização pelos atores envolvidos no processo de formação. O corpus de análise permitiu evidenciar que, em face do modelo tecnológico torna-se um suporte de ensino, uma ferramenta, um plug, por assim dizer, o processo de ensino-aprendizagem. O modelo (blended e-learning e) iminentemente virtual ou o ensino à distância é proposto como um paradigma de tecnologia educacional.
\end{abstract}

Palavras-chave: TIC, sala de aula, educação, faculdade, comunicação. 


\section{Introducción}

Las tecnologías de la información y la comunicación (TIC) son un concepto que proviene de dos vertientes: por un lado, las ciencias sociales, y por otro, la ciencia informática. A su vez, está constituido por la interacción de dos conjuntos de tecnologías: aquellas ligadas a la comunicación, conformadas por la radio, la televisión, la telefonía convencional, y las que están próximas a las tecnologías de la información, caracterizadas por la digitalización de los registros de contenido, como son la telemática, la informática y las interfaces. El mayor exponente de esta convergencia entre los dos conjuntos de tecnologías es el vertiginoso crecimiento de Internet, ecosistema, digital-comunicativo, que capitaliza los diferentes avances tecnológicos que ha logrado el ser humano a través de la historia y han repercutido en las formas de comunicación (escrita, sonora, visual); campo de las telecomunicaciones que ha propiciado no solo la correlación de medios y la integración estratégica de competencias profesionales, sino el surgimiento de un nuevo lenguaje cross-mediático y trans-mediático que impacta las actividades cotidianas del ser humano (Barrios, 2016), la forma de ver el mundo y la manera como se prepara para afrontarlo; un entorno que responde a las características de un entramado social ligado a conceptos tales como "tecnologías de la información"1, "sociedad del conocimiento"2 y "sociedad de la información"3.

Se está al frente de un contexto cultural y tecnológico desde el cual se gesta la reconstrucción social del mundo de hoy (Martín-Barbero, 2002). Los adultos y los jóvenes en el espacio digital propician un escenario de sociedad virtual en el que se establecen puntos de encuentro, se consolidan vínculos de amistad y se estrechan mecanismos de comunicación. La biosfera digital se constituye en el centro de información, pero, a su vez, en un ágora de aprendizaje donde se encuentra el pasado, lo aprendido y el dato específico que es útil; autopista del conocimiento que reduce el esfuerzo del ser y optimiza los procesos de búsqueda, dejando de lado una acción coactiva del proceso educativo tradicional.

Las TIC y el entramado social piden a la academia no ser considerados como "elementos exógenos" (Baelo \& Cantón, 2010, p. 166); es indispensable incorporarlas al proceso formativo, no de manera instrumental sino como mecanismo de reflexión y autocrítica del papel del sujeto frente a ellas. Evidenciar que proveen a las personas de herramientas prácticas para su desarrollo personal, laboral y académico (Barrios \& Zambrano, 2015), pero que el ser es superior a ella, no un objeto de la misma. De ahí la importancia de su

1 Término acoplado para hacer referencia a a los desarrollos más recientes dentro del campo de la comunicación, que incluyen desde la telefonía celular, los dispositivos portátiles, hasta la Internet, la tecnología digital, los desarrollos de la microelectrónica (Esteve Ramírez E Sandoval Martín, 2006).

2 "Se trata de una sociedad en la que las condiciones de generación de conocimiento y procesamiento de información han sido sustancialmente alteradas por una revolución tecnológica centrada en el procesamiento de información, la generación del conocimiento y las tecnologías de la información" (Castells, 2002, p. web).

3 "Se caracteriza por una serie de rasgos socioculturales, como la complejidad, la virtualidad, la globalización, la diversidad cultural y la centralidad de la presencia de las TIC" (Fainholc, 2002, p. web). 
culturización, uso y apropiación para favorecer la realización de procesos y proyectos que trascienden las barreras locales.

\subsection{El ecosistema digital en el proceso educativo}

En los últimos años, la educación en el mundo ha tenido cambios significativos, dentro de los cuales la implementación de nuevas tecnologías en el aula de clase ha sido el método innovador por excelencia para desarrollar la mayoría de los procesos educativos y de contacto entre docente y estudiante (Jump, 2011). Esta técnica ha sido avalada por algunas instituciones educativas como una buena manera de educar y de abrir espacios diferentes a sus estudiantes. La innovación tecnológica se convierte en una herramienta fundamental para los maestros en pro de optimizar, y ofrecer nuevas y mejores formas de enseñar, aprender y llegar al conocimiento (Dobozy, 2011).

La incorporación de las TIC genera una ruptura en el tiempo y el espacio de los escenarios educativos tradicionales. De la interacción cara a cara, punto de tiempo en un salón de clase en el que se ven profesor y estudiante para establecer una comunicación bidireccional de preguntas y respuestas, que enriquecen el proceso formativo, se da paso a una relación mediada por la tecnología que implica ya no solo compartir un escenario presencial en un momento determinado sino hacer uso de instrumentos como el teléfono móvil y la Internet, para ampliar los períodos de contacto e interacción, esquema comunicativo que se complementa con una interrelación casi-mediática que se da a través de los medios sociales (blogs, redes, wikis, entre otros), en los cuales los actores del proceso educativo están en horarios y áreas diversas, y la realimentación es colaborativa y se prolonga en los días (Fandos, 2008).

Las universidades apuestan por un uso de las TIC como apoyo a la presencialidad, intento de abordar el aprendizaje significativo a partir de los recursos y herramientas digitales e hipertextuales, modalidades educativas que son apoyadas con tableros, pantallas electrónicas o plataformas de carácter gratuito y software abierto como Moodle, de modo que se propende por la personalización de circuitos privados de interacción comunicativa y pedagógica, que se complementan con otros recursos de la web 2.0. Se suscita una formación crítica que propende por desarrollar ideas y pensamientos en los estudiantes, haciendo hincapié en los valores, para construir y afianzar la propia autonomía, en una nueva visión de la tecnología, que se encarga de construir otras formas de adquisición de saberes (Ferrari, Punie \& Brecko, 2013).

Es relevante establecer cómo es que cada uno de los actores se enfrenta a la tecnología, cómo la interpreta, cómo la concibe, para qué la usa, si la comprende y qué lectura tiene de ella, en el proceso enseñanza-aprendizaje (Tejedor, García-Valcárcel \& Prada, 2009). El aula, como contexto mediador de la interpretación de la realidad que hacen los sujetos, tiene una enorme importancia, pues el ambiente del aula está relacionado con otros contextos, es decir, en ella hay una proyección socio-cultural de lo que ocurre fuera de ella (Leal, 2009). El salón de clase dejó de ser una estructura física cerrada al mundo cultural y real que se trasladó al escenario tecnológico. 
Atrás quedó la educación ligada al modelo tradicional, aquella que omitía todo proceder de la pedagogía crítica, ya que el profesor estipulaba una relación vertical con el educando; se forma así un receptor de contenidos, y se eliminan la creatividad y el cuestionamiento. Hoy se estimula que el estudiante construya su personalidad desde las propias experiencias transformadoras de los valores y conocimientos. El docente, como agente mediador del proceso educativo entre las Instituciones de Educación Superior y los estudiantes, integra las TIC a su quehacer para fortalecer la calidad del proceso de enseñanza-aprendizaje, y propone actividades para que los universitarios, a partir de relaciones y prácticas, construyan su conocimiento.

Una pedagogía liberadora es, ante todo, conciencia, crítica y subversión, e invita a luchar por una sociedad mejor, a construir día a día la utopía (Freire, 2005). La crítica como eje articulador del proceso formativo permite descubrirnos como imperfectos y seres en construcción, invita a hacer conciencia de que en esta misión el ser humano es susceptible de cometer errores, pero también de poder hacer correcciones. El educador es una persona consciente que lleva a cabo la desligación de lo monótono, haciendo rupturas para una transformación. En el ecosistema educativo de hoy los únicos textos válidos, serios y con contenido no son los escritos; la sociedad actual tiende a convertir en hipertexto (el texto de la comunicación digital) toda la producción del conocimiento que nos ha construido como sociedad.

Las nuevas tecnologías, más allá de lo instrumental, se consideran nuevas maneras de agenciar el conocimiento en el aula, bondades que desde las modalidades educativas brindan un objeto de reflexión y apuestas investigativas de los que están implicados en ellas, pues "además de planteamientos integradores, es necesaria la precisión de unas metas y objetivos y disponibilidad de estándares e indicadores, prácticamente inexistentes en lo referido a la integración de las TIC..." (Vidal, 2006, p. 545). Los docentes y los estudiantes piensan y utilizan los recursos digitales en el proceso educativo, lo que conlleva contextualizar el sentido, la concepción, el uso y la cultura que hay detrás de la acción pedagógica mediada por los mismos, explorando las relaciones e interacciones en el aula de clase en las asignaturas que permiten la reflexión de los campos de formación profesional.

\section{Metodología}

La incorporación de las TIC en el proceso de enseñanza-aprendizaje obliga a ubicarse en el contexto de la sociedad de la información y el conocimiento que tienen como antecedentes: las concepciones, determinantes de las actuaciones en el aula; los centros educativos, aplicabilidad y uso de las TIC en el aula; los recursos educativos, TIC; el contexto Institucional, uso de las TIC en la Universidad objeto de estudio; y las sociedades informacionales, nuevas tecnologías, racionalidad y eficacia. Desde este escenario se desprende el interés de dar respuesta a la pregunta: ¿Cómo conciben el uso de las TIC en el aula desde las relaciones, las prácticas y los diseños, los docentes y los estudiantes 
de un programa de Comunicación Social-Periodismo, de Colombia, en dos momentos diferentes 2010 y 2014?

Para ello se estableció un proyecto de investigación (fases I-II) cuyo objetivo principal era establecer la concepción y uso de las TIC en el aula, desde las interacciones, experiencias y esquemas académicos. Posteriormente a ello se suma la necesidad de identificar las concepciones que sobre las TIC han construido los docentes, describir las relaciones que narran los docentes sobre las TIC y su conocimiento, detectar las prácticas de las TIC para el apoyo del ejercicio docente, caracterizar los diseños de las TIC, en el desarrollo de las asignaturas, y propiciar una reflexión del uso de las nuevas tecnologías en un programa de Comunicación Social-Periodismo cuya modalidad es presencial.

Al tener como objetivo la contrastación de resultados se hace necesario conservar la metodología empleada en la primera fase del proyecto, realizar un análisis de caso desde una perspectiva cualitativa, aunque tiene una primera fase de exploración con unos resultados cuantitativos. Es decir, se va a realizar una exploración sociodemográfica que posibilite el análisis etnográfico posterior, desde una perspectiva interpretativista.

Los docentes y los estudiantes piensan y utilizan las TIC en el proceso educativo, lo que conduce a contextualizar la concepción, el sentido, y el uso que hay detrás de la acción pedagógica mediada por las TIC, explorando las relaciones e interacciones en el aula de clase en las asignaturas que permiten la reflexión de campos de formación profesional. La población objeto se escogió teniendo como requisito, tener aprobadas las asignaturas del área de teorías y que sus currículos formativos integraran asignaturas del área de comunicación y sociedad, y comunicación y nuevas tecnologías, lo que garantiza los pre-saberes necesarios para comprender y dar respuesta a las preguntas de la encuesta diseñada para este grupo objeto de investigación (Fajardo, 2015).

Tabla 1. Sujetos objeto de estudio

\begin{tabular}{|c|c|c|}
\hline Sujeto & Fase / año / cantidad & \multicolumn{1}{c|}{ Rangos } \\
\hline \multirow{5}{*}{ Estudiantes } & Fase I / 2010 / 300 & $\begin{array}{l}\text { 14 y } 17 \text { años inclusive. Relativa cercanía con la formación } \\
\text { Básica Secundaria, la mayor dependencia de los adultos y } \\
\text { unos usos más informales de la tecnología, pero probable- } \\
\text { Fase II / 2014 / 450, también mayor dependencia de ella. }\end{array}$ \\
\cline { 3 - 4 } & & $\begin{array}{l}18 \text { y } 21 \text { años inclusive. Jóvenes de rango medio que están } \\
\text { mayormente impactados por los procesos universitarios y } \\
\text { el entorno digital del mundo hoy. }\end{array}$ \\
\cline { 3 - 4 } & $\begin{array}{l}\text { Más de 22 años. Tienen relativamente mayor independencia } \\
\text { de los adultos, pueden tener una percepción diferente del } \\
\text { uso de la tecnología, y en muchos caos, han tenido otras } \\
\text { experiencias universitarias que los alejan de ciertas formas } \\
\text { de ser del promedio de edades. }\end{array}$ \\
\hline
\end{tabular}




\begin{tabular}{|c|c|c|}
\hline Sujeto & Fase / año / cantidad & Rangos \\
\hline \multirow{3}{*}{ Docentes } & \multirow{3}{*}{$\begin{array}{l}\text { Fase I / } 2010 \text { / } 15 \\
\text { Fase II / } 2014 \text { / } 60\end{array}$} & $\begin{array}{l}30 \text { a } 40 \text { años. Puede presentarse una tendencia de inme- } \\
\text { diación al uso y consumo de las tecnologías que tienen los } \\
\text { estudiantes de la UJTL por su relativa aproximación con la } \\
\text { formación universitaria, unos usos informales de la tecno- } \\
\text { logía (Messenger, juegos, celulares, MP4, iPod, Memorias, } \\
\text { etc.) y probablemente encontremos una dependencia de la } \\
\text { tecnología. }\end{array}$ \\
\hline & & $\begin{array}{l}41 \text { a } 50 \text { años. Puede tener una percepción diferente del } \\
\text { uso de la tecnología y en muchos casos cuenta con otras } \\
\text { experiencias universitarias. }\end{array}$ \\
\hline & & $\begin{array}{l}\text { Más de } 51 \text { años. Al estar tan alejado de los estudiantes } \\
\text { generacionalmente hablando puede dejar ver casos excep- } \\
\text { cionales y una distancia marcada frente al uso y consumo } \\
\text { de las nuevas tecnologías. }\end{array}$ \\
\hline
\end{tabular}

Fuente: elaboración propia

Definido el objeto de estudio y la base conceptual bajo la cual se trabaja, se procedió al diseño de los instrumentos que permitieron hacer el trabajo de campo en el cual se obtuvieron los insumos cualitativos y el corpus de análisis cualitativo, elementos que fueron discriminados y organizados acuerdo con la información recolectada, lo que permitió su análisis e interpretación, para su posterior conceptualización.

Son escasos los estudios desarrollados en el ámbito educativo, y en tal sentido su propósito es la búsqueda de los significados que las personas otorgan a sus experiencias como miembros de una comunidad. De lo anterior se desprende que, en el proceso de estudio, se recurriera a la perspectiva interpretativista donde el investigador indagó y comprendió los fenómenos sociales de los actores, para desarrollar, y posteriormente exponer, óptimamente la conducta del hombre, recurriendo a los métodos de las ciencias sociales (Bouché, 2002).

El enfoque etnográfico enfatizó en los aspectos individuales y subjetivos de la experiencia, para de este modo descubrir lo que subyace a las formas, a través de las cuales convencionalmente las personas describen su experiencia desde las estructuras que las conforman. Fue un proceso en el que los investigadores propusieron preguntas, bajo unas categorías de análisis (Tabla 2), que permitieron explorar el significado de la experiencia al enfrentarse a las TIC en diversas situaciones de la vida cotidiana.

Tabla 2. Categorías de análisis

\begin{tabular}{|c|c|c|}
\hline Categoría & \multicolumn{1}{|c|}{ Qué busca } & \multicolumn{1}{c|}{ Elementos que incluye } \\
\hline \multirow{5}{*}{ Concepción } & $\begin{array}{l}\text { Encontrar en la propia mente razones o } \\
\text { explicaciones para cierta cosa. Acciones } \\
\text { congruentes con el pensamiento desde la } \\
\text { relación del "Yo" con el sujeto. }\end{array}$ & $\begin{array}{l}\text { Definición, pensamiento, aplica- } \\
\text { ción, implementación, uso, otras } \\
\text { tecnologías }\end{array}$ \\
\hline
\end{tabular}




\begin{tabular}{|l|l|l|}
\hline \multicolumn{1}{|c|}{ Categoría } & \multicolumn{1}{|c|}{ Qué busca } & \multicolumn{1}{|c|}{ Elementos que incluye } \\
\hline Práctica & $\begin{array}{l}\text { Qué hace el sujeto: docente-estudiante. } \\
\text { Cómo se apropia y explota la tecnología. } \\
\text { Prácticas de uso y consumo. }\end{array}$ & $\begin{array}{l}\text { Acción profesional, uso, formación, } \\
\text { rol, nivelación, mediaciones, ac- } \\
\text { ciones. }\end{array}$ \\
\hline Relaciones & $\begin{array}{l}\text { Interacción con y entre los sujetos a través } \\
\text { de las prácticas y el diseño }\end{array}$ & $\begin{array}{l}\text { Comunicación, definición, interac- } \\
\text { ción, recursos, elementos, uso de } \\
\text { TIC }\end{array}$ \\
\hline Diseño & $\begin{array}{l}\text { Cómo se debe estructurar la información y } \\
\text { la relación de comunicación para lograr el } \\
\text { objetivo en el receptor }\end{array}$ & $\begin{array}{l}\text { Formación, sociedad interactiva, } \\
\text { sociedad virtual, interacción, uso }\end{array}$ \\
\hline
\end{tabular}

Fuente: elaboración propia

En la investigación que ahora se expone, se recurrió al método etnográfico, centrando el estudio en el modo de vida del grupo en contextos educativos y sociales, emergiendo como un área propia de indagación y observación de los fenómenos de interacción que tienen lugar en dicho contexto a partir de diversas perspectivas (docente-estudiante). Se habla de un enfoque cualitativo, no explicativo, fundamentado en la palabra y no en el dato. Es fundamentalmente interpretativista, un proceso en el que se trabajó el todo y sus relaciones, bajo las categorías de análisis expuestas en la tabla 2.

Desde el enfoque cualitativo-interpretativo, la etnografía para la investigación, permitió dar cuenta de las lógicas de interacción y comunicativas de los actores del proceso de relación dentro y fuera del aula, mediado por las TIC. El uso y aplicación de sistemas y directrices del método etnográfico permitió a los investigadores realizar, a partir de una estructuración de actividades y una serie de acciones, enunciar y describir desde la perspectiva interpretativista, lo que sucede en un espacio y en un tiempo establecido, para así resolver los interrogantes, propuestos en el transcurso de la investigación (pregunta, objetivos, hipótesis).

En la metodología etnográfica, se abordaron diversas técnicas e instrumentos, que permitieron profundizar en la investigación, a partir de la recolección de datos, obtenidos en la aplicación de las técnicas seleccionadas para el proceso investigativo: la encuesta, aplicada al $100 \%$ de estudiantes en las dos fases, y la entrevista desde las cuales se desarrolla la observación científica.

\section{Resultados y discusión}

Es importante iniciar esta exposición de resultados abordando en primera instancia la encuesta para cerrar este apartado con la exposición de la entrevista y sus categorías de análisis.

La información etnográfica de este análisis cuantitativo lleva a referenciar que este instrumento fue aplicado a los estudiantes. Tanto en la primera (2010) como en la segunda (2014) fase de estudio se aprecia una mayor presencia femenina, acorde al programa que 
se está analizando (Comunicación Social-Periodismo) y a la media nacional que según el Departamento Administrativo Nacional de Estadística (DANE) refleja que en Colombia hay más mujeres que hombres. Al tratarse de una encuesta de público juvenil se observa una total participación de personas que oscilan entre el segundo y tercer rango de análisis (18 años o más), sin vínculos familiares (hijos) y ningún tipo de compromiso estable de pareja.

Frente a la competencia tecnológica, ha disminuido el aprendizaje empírico, sin embargo, en la segunda fase aún el 50 \% de los estudiantes manifiesta un conocimiento basado en el "cacharreo" y el 46 \% evidencia una mezcla de cursos luego del empirismo. Es evidente que la carga de instrucción en sistemas está concentrada en el Bachillerato, donde se responde a la expectativa de la Básica Primaria, pero desde la formación profesional no se potencia, capitaliza y profundiza en dichas competencias.

Preocupa que no hay un interés por la capacitación informática externa al medio universitario y llama a cuestionarse que los Comunicadores Sociales-Periodistas solo consideren importantes en su aprendizaje los programas básicos de Microsoft (Word, Excel y PowerPoint) dejando de lado los de edición de audio y vídeo; se rescata, que hay una noción de diseño de páginas Web, tal vez por la modificación curricular reciente del programa de formación. De ello se desprende que el dominio del lenguaje tecnológico sea básico; muy pocos (22 \%) se consideren avanzados y solo el 2 \%, expertos.

Desde la accesibilidad a la tecnología informática se percibe la penetración de la tecnología en los hogares de los estudiantes, pues del $9 \%$ que manifestaba no contar con computador en la primera fase, se pasa al $1 \%$ en la segunda. Igualmente se ratifica la importancia de la portabilidad del computador, pues se pasa del $7 \%$ de estudiantes con portátil a un $92 \%$ en la nueva etapa de estudio. Cobra cada día mayor importancia el uso personal y exclusivo del computador; de ahí que el acceso a la tecnología informática ya no se dé principalmente en la universidad, donde amigos y en el café Internet, como sí ocurría a comienzos de esta década. Asimismo, se logra entrever una fuerte penetración y masificación de los dispositivos portátiles (tabletas, celulares, entre otros.).

Con la llegada de los dispositivos portátiles, los planes de datos y las zonas Wi-Fi el acceso a la red es permanente y no está ya ligado únicamente a la permanencia en la casa, la universidad, el café Internet, el trabajo, las cabinas telefónicas, familiares y amigos, consecuencia evidente de los celulares de alta gama reflejada por el 78 \% de los encuestados en la segunda fase de estudio.

La percepción de uso permite apreciar un empleo múltiple del computador en actividades académicas, profesionales y lúdicas. Genera inquietud que, de un acercamiento constante a la herramienta tecnológica en la primera etapa de estudio, en la segunda se evidencie lo semejante que es la frecuencia de uso del computador: así como hay casos de extremos (diario), igual se observa la gente que manifiesta hacerlo mensualmente. La mayor frecuencia de uso del computador se va en un promedio de 1 a 3 horas, muy seguido de las personas que lo hacen en períodos de 4 a 6 horas. Lo anterior contrasta con la conexión a Internet que hoy en día supera las 7 horas al día, en diversos fragmentos, 
debido a su acceso a través del celular, un uso que se centra en actividades bastante lúdicas y alejadas a la educación.

Concordantes con la era digital todos dicen tener cuenta de correo electrónico, y el mayor cruce de correos se da con amigos y compañeros, marcando una distancia importante con padres y hermanos, y aún mayor con otros familiares. Se visualiza fácilmente que la relación docente estudiante cobra importancia a través del correo electrónico, el aula virtual y las redes sociales. Es interesante que en la primera fase de estudio el $50 \%$ de los encuestados se consideraba un usuario promedio, mientras que se reconocía como adicto el $8 \%$, y usuarios por necesidad el $18 \%$; llamaba a cuestionarse que el $25 \%$ no encontraba cómo calificarse. Ello contrasta con que en una época como la que vivimos, un $20 \%$ aún se refleje como usuario por necesidad, un $72 \%$ como usuario promedio, un $6 \%$ como adictos, y un $3 \%$ aún no sepa cómo calificarse. Los resultados anteriores contrastan con el hecho de que el $46 \%$ considere el PC como muy importante en su vida, y el $37 \%$, como importante. A ello se suma que el $23 \%$ se ve como muy conectado, el $43 \%$ se asume como conectado con la tecnología, y el 29 \% como medianamente conectado; solo un 5 \% no encuentra cómo definirse.

Lo hasta acá expuesto se complementa centrando la atención en las entrevistas y sus categorías de análisis que permiten decir:

Frente a la concepción, las TIC son consideradas por los docentes como herramientas, instrumentos, utensilios, elementos y recursos de apoyo para la comunicación e información, que son derivados de la tecnología digital actual, donde el principal propósito es el de facilitar el desarrollo del trabajo formativo y educativo, ya que permite adquirir, transmitir, compartir y generar contenidos a través de una red informática como Internet y mediante dispositivos electrónicos como el computador.

La concepción sobre las TIC depende también de las reflexiones que hacen los docentes para sustentar su definición y que tienen que ver con las ventajas o riesgos de su penetración en los distintos ámbitos de la vida. A pesar de que el grupo de docentes entrevistados señala que este tema es fundamental para el aprendizaje, también expresa su preocupación por el adecuado control que se les debe dar a estas herramientas, ya que consideran que puede ser desperdiciado o mal utilizado hasta el punto de llegar a ser perjudicial.

La categoría práctica se relaciona con la apropiación de las TIC en el aula de clase, en relación con los contenidos programáticos y objetivos formativos de las asignaturas; en este sentido estas juegan un papel en dichos escenarios; en relación con ello, algunos docentes entrevistados de Comunicación Social-Periodismo consideran que las TIC están presentes en todos los aspectos de la asignatura no solo como apoyo a la práctica académica sino porque se relaciona con la naturaleza de la misma. La presencia de las TIC es primordial para el desarrollo de cada asignatura, ya que el entorno profesional actual exige que los estudiantes no solo adquieran el conocimiento, sino que dominen las pla- 
taformas digitales, el software especializado, la integración de los recursos tecnológicos y la interacción en las redes sociales.

Los retos y obstáculos en el uso de las TIC son importantes para comprender la forma en la que los docentes hacen uso de ellas, pues consideran que, aunque son muy pocas las dificultades que se presentan, a menudo se resuelven con facilidad porque los jóvenes están muy involucrados con el manejo de diversos programas y tienen buena fundamentación. El tipo de problemas que se presentan tiene que ver con el dominio técnico de un software o el manejo de la plataforma y se suelen solucionar con el diálogo, la práctica repetitiva para memorizar las funciones del mismo o a través del trabajo en equipo con el apoyo mutuo de los que manejan mejor el tema.

La práctica exige cierto nivel de conocimiento entre los docentes y estudiantes en los escenarios formativos que puede ser o no simétrica; de acuerdo con la mayoría de las respuestas la nivelación es necesaria para los docentes y no tanto para los estudiantes. Todos los entrevistados concuerdan en declarar la necesidad de hacer capacitaciones progresivas y de actualización especialmente para los docentes que vienen de una práctica tradicional. Se mencionan los siguientes temas relativos al uso de las TIC: manejo de ayudas audiovisuales, transmisión de la información y conocimiento mediado por TIC, la forma de hacer una presentación y cómo manejarla con fines pedagógicos.

La compresión del uso práctico de las TIC en los escenarios de clase debe tener correspondencia con la importancia que los docentes les conceden en los logros de los objetivos formativos; en relación con dicha importancia la percepción de los docentes entrevistados varía en función de la asignatura que les corresponde. Para algunos comunicadores el porcentaje de uso de las TIC en la formación de los estudiantes es alto incluso de un 90 o 100 \% en el plano social, ya que observan a los jóvenes inmersos dentro del ambiente tecnológico.

En la categoría relaciones se parte de reconocer propiedades en las estrategias apoyadas en las TIC que los actores de los procesos formativos validan en sus interacciones. En tal sentido, los entrevistados concordaron en nombrar como elementos esenciales de una pieza digital principalmente a un concepto, unos objetivos, un buen funcionamiento, un fácil manejo, una buena presentación y cumplimiento con lo que se propone. Además, que sea corto, preciso, conciso, sencillo, limpio, contundente y coherente.

En las relaciones es importante el reconocimiento de los métodos y técnicas comunicativas más apropiadas en el uso de las TIC en el aula; en este sentido los docentes entrevistados señalan que, a través del diálogo con los estudiantes y los profesores, la evaluación de las opiniones y la identificación de las respuestas se puede demostrar que la interacción académica y los objetivos se cumplen cuando el aprendizaje es logrado.

En la práctica es determinante poder analizar las condiciones que rodean el uso de las TIC en el aula de clase. La percepción que tienen todos los docentes entrevistados es que los estudiantes utilizan las TIC en la medida en la que el profesor de la asignatura 
los induce a hacerlo. Aunque para los jóvenes es natural el uso de la tecnología, como herramientas de estudio carecen de la visión necesaria para aprovecharlas al máximo y por eso es fundamental la guía del docente para estimularlos a que las conozcan y usen. Debido a eso, las falencias que pueda tener un docente en este campo se verán reflejadas en la interacción académica del joven.

Según los participantes, el recurso teórico abarca la construcción del contenido intelectual o conocimiento que se va a transmitir, y el práctico envuelve todos los elementos técnicos que son necesarios para la creación de la pieza diseñada. El recurso teórico encierra el discurso, la temática, el mensaje, mientras que el segundo contiene las herramientas digitales que, además de tener los programas informáticos que son necesarios para crear la producción como por ejemplo procesadores de texto, programas de presentación, diseño, edición, audio, animación, creación audiovisual, entre otros, también incluye los dispositivos necesarios para manejarlos.

Desde la categoría diseño, algunos docentes piensan que los jóvenes no necesitan de una formación previa para el uso de estas plataformas, ya que vienen de una generación educada con medios tecnológicos y saben desplazarse a través de diversas estructuras informáticas. Lo que sí necesitan son conocimientos específicos para entender ciertos procesos cognitivos y la aplicación de estos en el ámbito profesional; estas condiciones se pueden analizar en las entrevistas a partir de la categoría de formación para el uso. Los objetivos de los procesos de aprendizaje como leer, analizar, reflexionar no han mejorado; es más, la gran cantidad de información con la que cohabitan los estudiantes a diario les impide asimilar, ser críticos, sensibilizarse frente a lo que no comprenden y están acostumbrados a lo que es rápido, fácil e inmediato.

La sociedad interactiva en la que lo característico es que haya una acción comunicativa completa de todos los interlocutores no es cierta. Declaran que es equivocada la noción que se tiene de interactividad cuando se entiende como una participación unilateral en las redes sociales que no produce transformaciones y se vuelve únicamente informativa.

En la comunicación digital, la comprensión de la hipertextualidad es indispensable para intervenir en el diseño de los productos digitales. La mayoría del grupo de docentes definió lo hipertextual como una relación entre el texto y lo visual con la utilización de vínculos y rutas para transportar al usuario a otros escenarios de conocimiento, enmarcados por el uso de recursos tecnológicos.

\section{Conclusiones}

Las Universidades se encuentran en un proceso de actualización constante de recursos que les permitan garantizar calidad y eficiencia en sus actividades académicas, responder a los estándares internacionales de la educación, establecer escenarios de capacitación e información en donde no solo se trata de crear aulas virtuales por el solo hecho de tenerlas sino interiorizar y difundir una serie de procesos diferentes; los tipos de comunicación, 
los recursos y los grupos a los que está enfocada la docencia son heterogéneos, así como las herramientas que se utilizan para relacionarse con esos grupos.

Las plataformas virtuales se han incorporado al derrotero del constructivismo social determinado por el Proyecto Educativo Institucional de la Universidad. Impera el modelo de resolución de problemas, que es el estilo representativo de la enseñanza a través de la búsqueda, en el que el estudiante es protagonista del proceso de enseñanza-aprendizaje, pues los recursos se orientan a la ejecución de actividades. Sin embargo, la reflexión y planificación de la concepción y uso de las TIC en el aula han sido escasas, sobre todo en los aportes a los procesos académicos que se relacionan con la modalidad de apoyo a la presencialidad. El modelo de uso y apropiación de las TIC es de campus presencial con elementos TIC de apoyo, que combina metodologías y didácticas pedagógicas en un mismo ambiente de aprendizaje.

Las nuevas tecnologías indudablemente han transformado "las sensibilidades" para incluir un término que utiliza Martín Barbero (2002). Estas nuevas sensibilidades han alterado la forma de vida del ser humano y eso obliga a dejar atrás el calificarlas o el intentar valorarlas positiva o negativamente; indudablemente tienen unos alcances positivos muy buenos, pensando incluso en términos democráticos, pero también hay que entender que su uso no deja de ser también factor de exclusión. La relación con estas nuevas tecnologías es natural; es un asunto en donde casi el término que se puede emplear es de naturalización de la técnica, porque ya no es nada novedoso ni sentarse al computador, ni utilizar las nuevas tecnologías, pues hace parte del cotidiano de la vida.

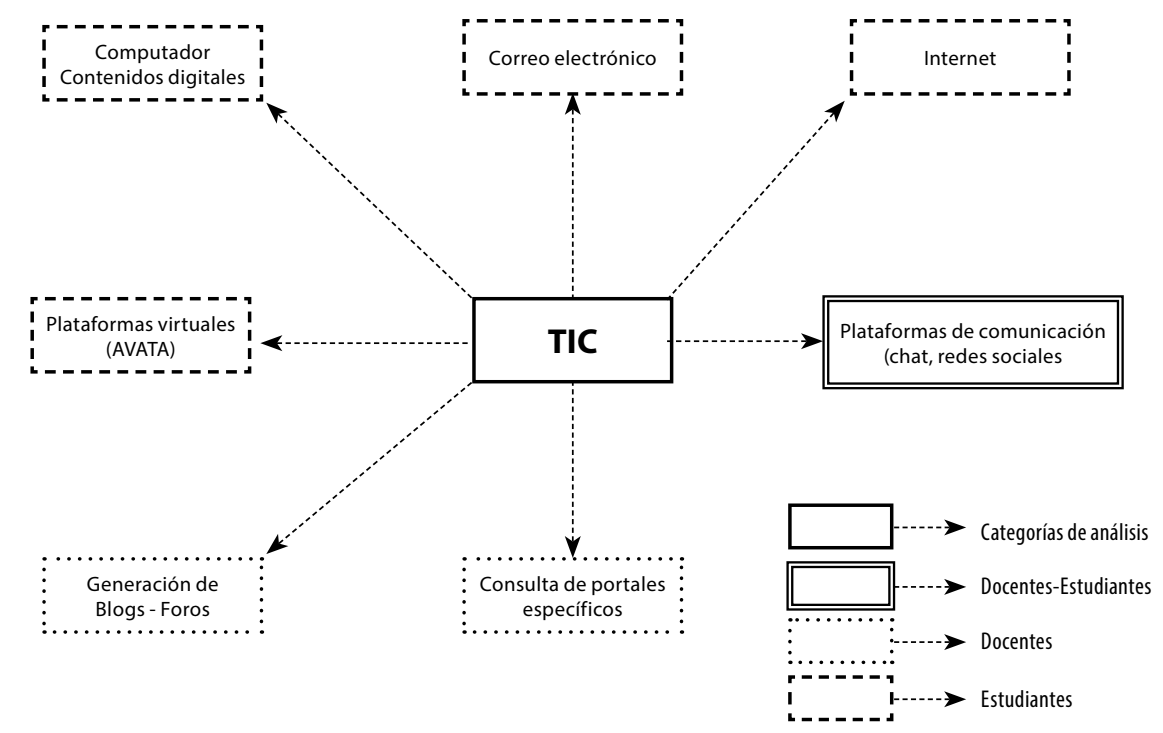

Gráfico 1. Referencias a "TIC" por parte de docentes y estudiantes Fuente: elaboración propia 
Las TIC como un conjunto de herramientas y recursos tecnológicos al servicio de la comunicación e información pueden ser utilizadas en diversos espacios de la vida cotidiana, cuyo propósito en el ámbito educativo es el de facilitar el acceso a la generación de contenidos. La importancia de estos instrumentos radica en la transformación que ha sufrido la manera de comunicarnos, la cantidad de conocimiento que puede ser acumulado en una red como Internet, la visión de la sociedad y los cambios en las dinámicas laborales, sociales, económicas, políticas, culturales y educativas que se han dado. Como consecuencia, la actuación de los sujetos está determinada por esta transformación y los procesos de enseñanza deben reflejar este cambio. El profesor se convierte en tutor-orientador y el adecuado uso de estas herramientas puede mejorar sus estrategias pedagógicas.

La brecha generacional que existe entre los actores del ambiente educativo está en el marco de un proceso natural y cultural en los individuos; es un distanciamiento de edad y conceptual que no lo da la tecnología, ni el uso de las mismas, sino las reconstrucciones y resignificaciones que cada una de las generaciones hace. Desde el principio de la humanidad han existido brechas generacionales porque eso hace parte de los procesos de desarrollo del hombre; es decir, hay un momento en la vida en que, independientemente de lo tecnológico, las posturas frente a un tema o situación son distintas, las reconsideraciones difieren, las maneras de ver el mundo de un ser son distintas a la de otros y eso es lo que genera una brecha generacional.

Se denota, en el proceso investigativo, un gran interés por parte del docente en superar el dominio de las nuevas tecnologías frente al estudiante, exigiéndoles retos en el aprendizaje e interés en el conocimiento en cuanto a su uso, aplicación y concepción de las TIC, y su relevancia frente al proceso educativo en el aula. No obstante, hay que reconocer que existen docentes que no se preocupan por estar a la vanguardia de las TIC y prefieren escudar su desconocimiento argumentando tres aspectos: el primero de ellos, la falta de tiempo, pues aparte de las clases está dedicado a otro tipo de actividades profesionales y académicas; el segundo, la capacidad económica y el valor de los cursos de capacitación; y el tercero, y último, el temor a "meterse" en el uso de las TIC porque consideran que el tener un aula virtual implica doblar el trabajo, asumir funciones adicionales a las ya establecidas en la presencialidad.

El análisis del corpus investigativo permite deducir que los jóvenes se sienten más atraídos por una pedagogía que incluya el uso de propuestas audiovisuales diversas en el proceso de aprendizaje. De igual manera, depende del docente incentivar la aplicación de los recursos tecnológicos en el desarrollo de la formación de los estudiantes, pero, si bien el uso de estas herramientas ofrece apoyo, no es lo esencial en el desarrollo de los ejercicios en el aula cuyo propósito final es el aprendizaje. La orientación del profesor es fundamental para encaminar la interacción académica de los jóvenes hacia la adquisición de su propio conocimiento. 


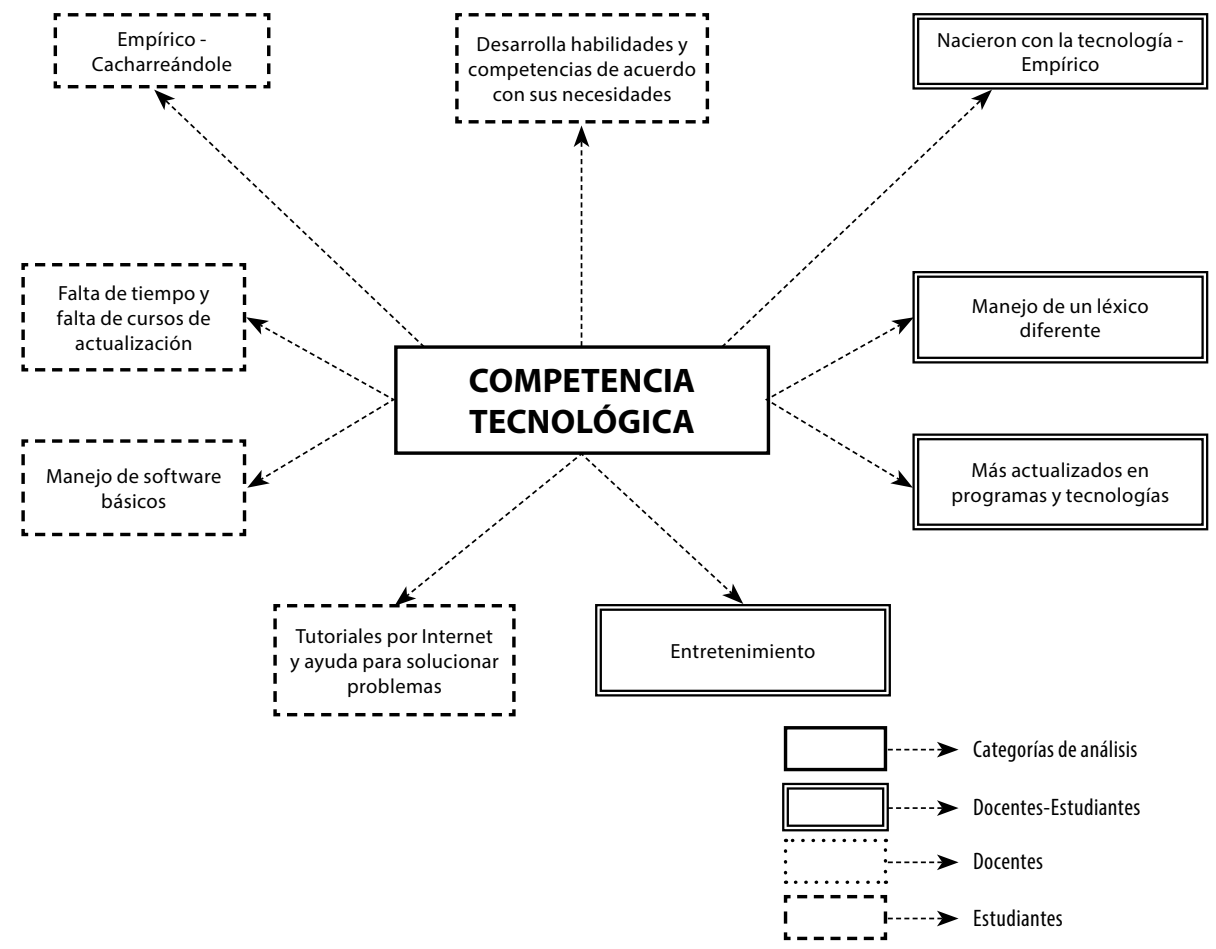

Gráfico 2. Referencias a "Competencia Tecnológica" por parte de docentes y estudiantes Fuente: elaboración propia

Las TIC no son ni positivas ni negativas en la formación de los estudiantes, la optimización de los procesos de aprendizaje depende de la intención con que se utilice, la manera como se aborda. La tecnología se convirtió en un referente social, y de poder adquisitivo. Su uso es fragmentario; es obligatorio reorganizar los procesos de pensamiento; la tecnología exige un pensamiento tecnológico, y no de un proceso de consumo cultural. Es relevante que las instituciones de Educación Superior recapaciten frente a la responsabilidad de educar y capacitar a los estudiantes y docentes en el uso y consumo de las nuevas tecnologías de la información y comunicación, TIC; es importante tener conocimiento y destrezas en el dominio de las diversas aplicaciones de las herramientas de comunicación y de las redes sociales, con el objetivo de fortalecer el uso y su impacto en el proceso educativo en el aula.

Dentro del marco educativo, las TIC son indispensables en aquellas materias que exigen conocimientos especializados en recursos tecnológicos para el desempeño profesional, a diferencia de aquellas temáticas, teóricas, que pueden ver limitada su aplicación al uso de una plataforma o algunos intercambios de comunicaciones digitales. Muchas posibilidades son desaprovechadas, al no percibir de manera directa la extensión de uso de estos recursos, o la carencia de las competencias técnicas necesarias que permiten 
integrar estos instrumentos en las prácticas académicas. La presencialidad y la virtualidad en la educación moderna se complementan y en ningún momento la una sustituye a la otra.

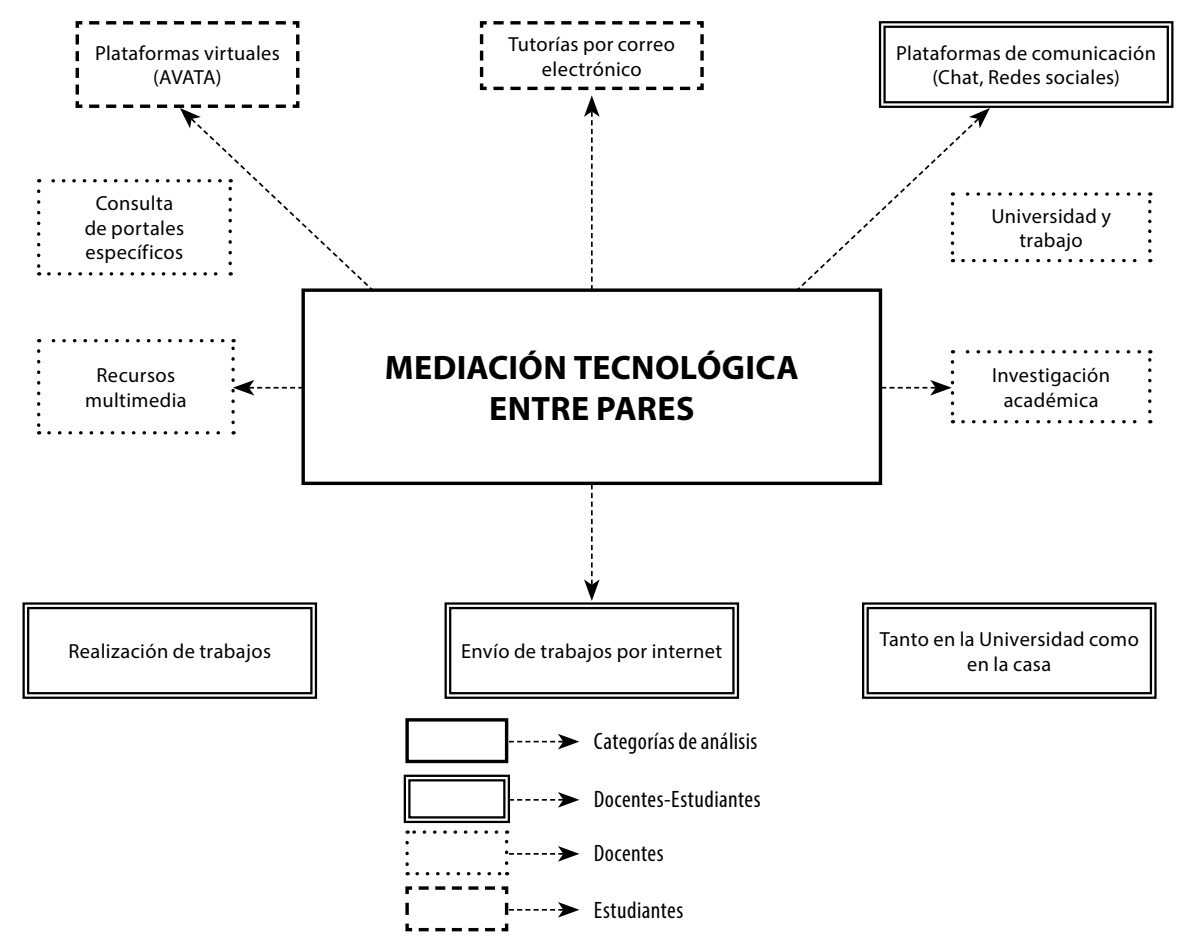

Gráfico 3. Referencias a "Medicación tecnológica entre pares" por parte de docentes y estudiantes Fuente: elaboración propia

A pesar del conocimiento y las destrezas de los estudiantes frente a las TIC, su uso en el aula es pobre, su reto está en enfrentar la pereza, de hacer uso de esa experticia con que cuenta, no solo limitarse al conocimiento superficial de los programas básicos, (Word, Excel, Power Point). El desafío está en indagar, ir más allá, experimentar, proyectar una imagen profesional, preocuparse más por ser mejor que sus pares, proponer ideas frente al reto del conocimiento, desarrollar e innovar a partir de sus intereses conceptuales. El estudiante debe cuestionarse constantemente el para qué: lo necesito, lo comprendo, es importante, lo encuentro, lo desarrollo, lo hago. La tecnología en el estudiante forma parte de los procesos de consumo, y no de un proceso cognitivo.

La gran apuesta de la cibercultura, en el plano del acceso de todos a la educación no está tanto en el paso de lo 'presencial' a la 'distancia' ni de lo escrito y oral tradicional a lo multimedia (Lévy, 2007), sino en la transición entre la educación de una formación estrictamente institucionalizada (la escuela, la universidad) a una situación de intercambio generalizado de los saberes, de enseñanza de la sociedad por ella misma, de reconocimiento auto dirigido, móvil y contextual de las competencias. Se requiere un uso racional y 
planificado de las TIC, que conciba la participación reflexiva de los actores involucrados en los procesos formativos. La incorporación de las TIC en el proceso académico trae consigo un cambio en la relación docente-estudiante dentro y fuera del aula de clase (gráfico 4).

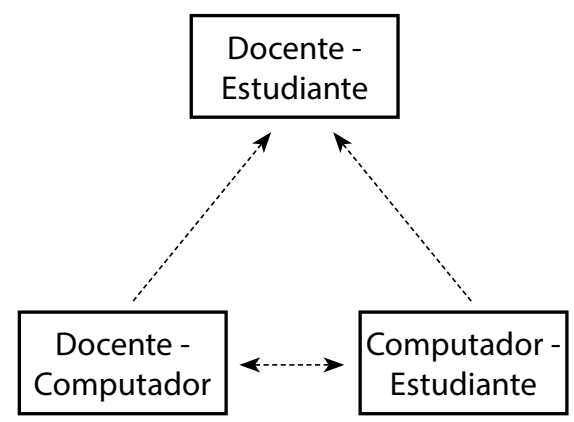

Gráfico 4. Nueva relación en el entorno pedagógico

Fuente: elaboración propia

Se transforma la relación de la educación y la comprensión de la educación. Este nuevo esquema hace que la comunicación fluya más fácilmente entre los actores del proceso educativo y se vuelva un poco más impersonal. Es un equilibrio entre la institución y quienes la integran, pero la iniciativa, la organización, la planificación tienen que venir de la institución y no del docente, pues quien determina los parámetros que se van a aplicar y la modalidad formativa a emplear son las universidades y los programas académicos aprobados por el estado. Es un proceso en el que los docentes deben ser conscientes de que las modalidades, las estrategias de enseñanza-aprendizaje han cambiado.

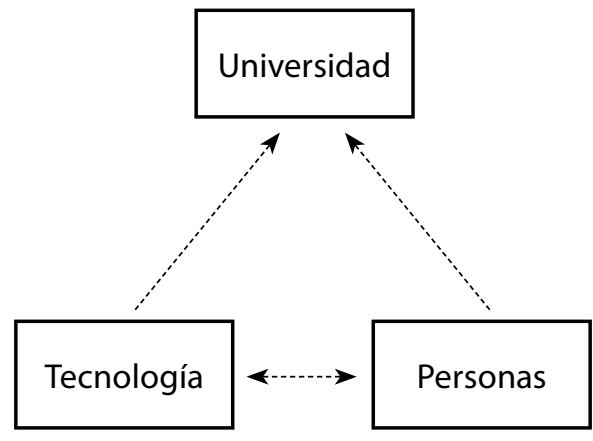

Gráfico 5. Tríada Universidad-TIC-Personas

Fuente: elaboración propia

Las universidades, en concordancia con los lineamientos del Consejo Nacional de Educación Superior de Colombia (CESU), enfocan todos sus esfuerzos a estructurar políticas para el impacto de las TIC en la academia, denominada como educación dual, es decir, de apoyo a la presencialidad. Se considera que la implementación de las modalidades 
de distancia o semipresencial (learnign o blended) han resignificado el papel del docente y del estudiante, y el diseño didáctico ha provocado la participación de nuevos actores en el proceso formativo, lo que también ha tenido influencia en la creación de nuevos ambientes de aprendizaje que se basan en formas diferentes de interacción mediadas por las TIC.

En Colombia existe la necesidad de establecer acuerdos y políticas para garantizar la calidad de los procesos formativos mediados por las TIC y sobre el deber que tienen las instituciones, en general, de propiciar la formación de competencias tecnológicas para que los estudiantes y profesionales estén en capacidad de interactuar con el conocimiento en un mundo globalizado, tarea en la que la incorporación de las TIC en el aula es fundamental (Consejo Nacional de Educación Superior, 2014). Sin embargo, se aprecia hasta el momento la falta de parámetros propios de las modalidades educativas soportadas en tecnologías digitales, así como falta de claridad conceptual con respecto a las implicaciones formativas.

La escasa investigación que existe del impacto del ecosistema digital en el entorno educativo no ha permitido consolidar y mejorar la implementación de las modalidades learnign o blended. Por ello desde este trabajo de investigación se resalta la necesidad de que exista una reflexión acompañada desde lo institucional sobre el uso, las prácticas y las relaciones formativas que se derivan del uso de las TIC en los procesos formativos, para racionalizar tanto los recursos como los objetivos de la formación por competencias.

Hasta el momento, como se evidencia en los resultados de este texto, estas apuestas están motivadas, por los intereses personales, tanto de los docentes, como de los estudiantes, y el uso de las TIC más que fortalecerse se aprovecha de estas bases, sin que haya una verdadera comprensión y coherencia con respecto a las condiciones de los espacios formativos. En otras palabras, a estos ambientes de aprendizaje virtual les hace falta la lógica y organización que se les exige a los ambientes o asignaturas de naturaleza presencial, condiciones que probablemente han afectado las iniciativas de la propia universidad para generar otras modalidades de aprendizaje basadas en las TIC.

En un contexto que parece saturado de información sobre la definición de estas modalidades, la reflexión que verdaderamente importa es la de concepción y uso racional de estos recursos por parte de todos los actores involucrados en los procesos formativos; puesto que es este el que garantiza los procesos de mediación, necesarios para las transformaciones de los escenarios y prácticas sociales, debido a que las mediaciones son, esencialmente, espacios y formas de uso de los bienes y recursos culturales, entre estos las TIC. Así entendidas, son las mediaciones las que transforman los escenarios sociales y culturales, y las que resignifican las relaciones y proveen respuestas a las problemáticas y necesidades de los actores sociales. 


\section{Bibliografía}

Baelo, R E Cantón, I. (2010). Las TIC en las Universidades de Castilla y León. Revista Comunicar, XVIII (35), 159-166.

Barrios, A. (2016). Narrativa periodística en la convergencia de medios. Estudios sobre el Mensaje Periodístico. 22 (1), 163-176. DOI: http://dx.doi.org/10.5209/rev_ESMP.2016.v22.n1.52587

Barrios, A.; E Zambrano, W. R. (2015). Convergencia digital: Nuevos perfiles profesionales del periodista. Revista Anagramas. 14 (26), 221-240.

Bouché, H (2002) La etnografía en el aula. En Bouché, H. et al. (2002) Antropología de la Educación. Madrid: Síntesis.

Consejo Nacional de Educación Superior. (2014). Acuerdo por lo Superior 2034. Bogotá- Colombia.

Dobozy, E. (2011). Typologies of Learning Desing and the Introduction of a LD-Type 2 Case Example. eLearning Papers. N. ${ }^{\circ} 27$.

Esteve Ramírez, F. \& Sandoval Martín, M. T. (2006). Luces y sombras de las nuevas tecnologías de la información. Ámbitos, (15), 33-44.

Fajardo, G. C. (2015). Apropiación de las TIC: concepción, uso, relaciones, prácticas y diseños en el aula (Caso programa Comunicación Social-Periodismo de la Universidad de Bogotá Jorge Tadeo Lozano). Universidad Santo Tomás. Bogotá-Colombia.

Fandos, M. (2008). El reto del cambio educativo: nuevos escenarios y nuevas modalidades de formación. Educar, (38), 243-258.

Freire, P. (2005). Pedagogía del oprimido. 2. ${ }^{\text {a }}$ edición. México: Siglo XXI Editores S. A. de C. V.

Jump, L. (2011). Why University Lectures Enhance their Teaching through the Use of Technology. A Systematic Review. Learning Media and Technology, 36 (1), 55-68.

Leal, A. (2009). Introducción al discurso pedagógico. Horizontes Educacionales, 14 (1), 51-63.

Lévy, P. (2007). Cibercultura, la cultura de la sociedad digital. Barcelona: Anthropos Editorial.

Martín-Barbero, J. (2002). La educación desde la comunicación. México: Gustavo Gili.

Tejedor, F. J.; García-Varcárcel, A. E Prada, S. (2009). Medida de actitudes del profesorado universitario hacia la integración de las TIC. Revista Comunicar, XVII (33), 115 124.

Vidal, M. P. (2006). Investigación de las TIC en la educación. Revista Latinoamericana de Tecnología Educativa, $2(5), 539-552$.

\section{Referencias digitales}

Castells, M. (2002). La dimensión cultural en Internet. En Institut de Cultura, Debates Culturales. Universitat Oberta de Catalunya. Recuperado el 10 de julio de 2015. Disponible en http://www. uoc.edu/culturaxxi/esp/articles/castells0502/castells0502.html

Fainholc, B. (2002). El concepto de Mediación en la tecnología Educativa Apropiada y Crítica. Buenos Aires, Ficha Cátedra UNLP. Recuperado el 10 de julio de 2015. Disponible en http://www.cediproe. org.ar/producciones 
Ferrari, A.; Punie, Y. \& Brecko, B. (2013). DIGCOMP a Framework for Developing and Understanding Competence in Europe. En: Joint Research Centre $\mathcal{E}$ Institute for Prospective Technological Studies. Recuperado el 10 de julio de 2015. Disponible en http://ipts.jrc.ec.europa.eu/publications/pub. cfm?id=6359 